\title{
IL DOLORE, GLI OPPIACEI E I PROBLEMI NEL LORO USO
}

\author{
ALBERTO PANERAI (*)
}

\begin{abstract}
SunTO. - Negli ultimi anni l'uso di farmaci oppiacei è andato costantemente aumentando. In Italia il loro impiego è sempre stato molto limitato, forse per ragioni culturali, forse per le limitazioni di legge nel loro uso, detenzione e trasporto, forse per il timore del medico di effetti collaterali o il timore di indurre tossicodipendenza. Recentemente gli aspetti legislativi sono stati superati e in parte anche i timori di altra origine. L'aumento dell'uso degli oppiacei è un fenomeno mondiale, più evidente negli Stati Uniti dove, dopo un periodo di grande entusiasmo e incremento, sono iniziati a sorgere diversi dubbi che stanno innescando un processo inverso, con la raccomandazione di usare sì gli oppiacei, ma stando molto attenti a come e in quali pazienti. Questo aspetto "sociale" dell'impiego di oppiacei, si accompagna poi al manifestarsi di una serie di problemi prima sconosciuti, che ne rendono l'impiego meno semplice del previsto.
\end{abstract}

$* * *$

ABSTRACT. - Several reasons recently lead to a dramatic increase in the use of opiate analgesics all over the world. Italy has always been a Country where opiates were prescribed less than in other Countries, even those incorrectly called "under-developed Countries". The reason has to be searched in cultural, regulatory (detention, transport) factors, as well in the caution of practitioners for the side effects of opiates or the fear of inducing addiction. Recently rules have become easier also in Italy and here, as well as abroad, mainly in the United States, the use of opiates has been rapidly increasing. However, the enthusiasm was followed by several serious doubts that are prompting a reversal of this trend. At present, the suggestion is to use opiates, but to pay a lot of attention to when use them and in which patients. Moreover, this "social" caution, was matched by the observation of a number of problems before unknown that make the use of opiates more difficult than it was previously thought.

(*) Dipartimento di Scienze Farmacologiche e Biomolecolari, Università degli Studi di Milano, Italia. E-mail: alberto.panerai@unimi.it 


\section{PREMESSA}

Una breve premessa riguardo al rapporto tra il Professor Mantegazza e la farmacologia del dolore. Non credo che lo studio del dolore, della sua fisiopatologia o terapia sia mai stato un interesse primario del Professor Mantegazza. Eventualmente l'interesse del Professor Mantegazza poteva essere attratto dalle singole molecole e la relazione struttura effetto, come del resto era per qualsiasi tipo di molecola/farmaco: il Professore era soprattutto un farmacologo "chimico", a differenza dei farmacologi attuali che sono farmacologi "funzionali", non sempre interessati alla struttura della molecola che stanno usando.

L'esame delle pubblicazioni del Professor Mantegazza conferma questa visone: considerando la lunga carriera scientifica, solo nel 1973 compare un lavoro che, seppure marginalmente, riguarda il dolore. Il lavoro successivo che in qualche modo possa essere ricondotto ai farmaci analgesici è del 1980 [1] e da allora seguiranno tra il 1980 e il 1991 alcuni lavori [2-8] in collaborazione con la Professoressa Nerina Zonta riguardanti la modulazione dell'attività della morfina.

Il nome del Professore è presente dagli anni ' 80 in molti lavori del nostro gruppo relativi alla fisiologia e terapia del dolore, ma soprattutto per il suo insostituibile supporto umano e anche economico. L'unico lavoro cui partecipò attivamente e lo interessò fu un lavoro che, confermando quanto detto sopra, riguardava la possibilità di modificare gli effetti analgesici e immunomodulatori della morfina modificando la sua struttura chimica [9].

\section{IL DOLORE}

La I.A.S.P. (International Association for the Study of Pain) definisce il dolore: "un'esperienza sensoriale ed emozionale spiacevole associata a un danno tessutale effettivo o potenziale" riunendo sotto un grande ombrello tanti tipi di dolore, con cause e terapie diverse. Con una buona semplificazione, possiamo riconoscere tre tipi di dolore. Il dolore nocicettivo è quello che segue a uno stimolo diretto (una martellata) e permane per il tempo di durata dello stimolo per poi diminuire e terminare. Il dolore infiammatorio parte dalla periferia ed è iniziato dalla presenza di diversi fattori algogeni come bradichinina, istamina, serotonina, prostaglandine che caratterizzano appunto l'infiammazione e termina con il ter- 
minare dello stimolo. Il dolore più complesso e che pone i più rilevanti problemi di fisiopatologia e terapia è il dolore neuropatico. Questo può derivare da una lesione delle vie di conduzione nel sistema nervoso centrale o periferico o da una loro disfunzione. Il problema di questo dolore, le cui basi sono in gran parte ancora sconosciute, risiede nel fatto che permane dopo la risoluzione dello stimolo e diventa un dolore cronico. Due sintomi particolari caratterizzano il dolore neuropatico: l'iperalgesia, cioè un aumentato senso del dolore in risposta allo stimolo nocicettivo e l'allodinia, cioè un dolore derivante da uno stimolo che normalmente non produrrebbe dolore. Ultimo problema del dolore neuropatico è la scarsezza di farmaci attivi e la loro scarsa e temporanea efficacia e anche gli oppiacei sono scarsamente o per niente attivi.

Come detto, non si sa molto della fisiopatologia del dolore neuropatico, ma nuove e forse inattese conoscenze ci stanno aprendo a un ulteriore livello di complicazione, ma anche di comprensione di questa patologia, con la possibilità di nuovi approcci terapeutici. Negli ultimi anni si è infatti dimostrato con certezza che il sistema immunitario gioca un importante ruolo nella genesi e mantenimento di questo dolore (si è visto poi anche altri tipi di dolore), con la attivazione microgliale e la secrezione di citochine pro-infiammatorie come interleuchina-1 (IL-1), Interleuchina-6 (IL-6), tumor necrosis factor alfa (TNFalfa) e diminuzione delle citochine anti-infiammatorie come interleuchina-4 (IL-4), Interleuchina-10 (IL-10). Di fatto, si è anche osservato che il capovolgimento di questo equilibrio a favore delle citochine anti-infiammatorie si accompagna alla remissione della patologia [10].

In conclusione possiamo parlare di un dolore "fisiologico": nocicettivo, segnale d'allarme, fenomeno riflesso, senza allodinia e iperalgesia, limitato nel tempo, e di un dolore "patologico": clinico, caratterizzato da una sensibilizzazione spinale, iperalgesia e allodinia.

Una recente e importante acquisizione è che il dolore cronico, qualunque sia la sua origine, per la sua gravità viene oggi considerato non più un sintomo come il dolore nocicettivo e quello infiammatorio, ma una malattia a sé.

\section{GLI OPPIACEI}

I primi farmaci oppiacei derivano dal lattice del papavero sonnifero e sono tre: la morfina, la codeina e la tebaina, con una struttura molto 
simile tra loro. Attorno a questa struttura si è lavorato molto nell'intento di ottenere nuovi farmaci che avessero qualche vantaggio in termini di potenza, effetti collaterali, durata di azione rispetto alle molecole naturali. Si sono così ottenuti gli oppiacei semi-sintetici (eroina, ossimorfone, ossicodone, destrometorfano, buprenorfina, tramadolo), sintetici (metadone, meperidina, fentanil) e dei farmaci antagonisti (naloxone, naltrexone). Su quale vantaggio questi abbiano portato rispetto alla morfina in termini di effetto analgesico in rapporto all'uso terapeutico la discussione è assolutamente aperta. Di fatto tutti gli oppiacei hanno gli stessi effetti sia desiderati (analgesia, antitosse) che collaterali/avversi (depressione respiratoria, stipsi, emesi, nausea). L'unico effetto, clinicamente forse insignificante, nel quale mostrano qualche differenza è nella modulazione del sistema immunitario sul quale agiscono generalmente come depressori, mentre metadone e buprenorfina non sembrano avere effetto e il tramadolo sembra avere un effetto stimolante che, tuttavia, non deriva dalla sua azione come oppiaceo. In realtà credo che si possa dire che la ricerca di nuove molecole ad effetto oppiaceo non abbia portato a molto e che, malgrado gli sforzi scientifici e commerciali, sia ferma o proceda senza evidenti progressi.

\subsection{Avanzamenti nell'uso degli oppiacei}

La ricerca non si è rivolta solo alla individuazione di nuove molecole, ma anche a migliorare l'uso degli oppiacei e la loro tollerabilità.

La scoperta negli anni '70 degli oppiacei endogeni (endorfine, encefaline, dinorfina) ha portato alla scoperta anche di recettori oppiacei che si manifestano solo in tessuti infiammati [11]. La conseguenza di questa scoperta è stata la possibilità di utilizzare questi farmaci anche per una analgesia periferica, mai prima sospettata per gli oppiacei, somministrandoli ad esempio per via intra-articolare. Per questa via, si possono usare alte concentrazioni di farmaco, senza che si manifestino evidenti effetti collaterali sistemici: per questa ragione la pratica è ormai di uso comune.

Un altro importante passo avanti nell'uso degli oppiacei è stato il superamento del problema della stipsi. Tutti gli oppiacei inducono contrazione della muscolatura liscia intestinale e così rallentano la peristalsi, portando a stipsi. Questi effetti, benchè abbiano anche una componente centrale, sono soprattutto mediati dal legame ai recettori oppiacei presenti sulle pareti intestinali e quindi più evidenti con l'uso di farmaci 
per somminsitarzione orale. Inoltre, lo sviluppo di tolleranza è molto lento o non si manifesta. Questo aspetto è cruciale, perché mentre la depressione respiratoria, apparentemente un effetto molto grave, nella pratica clinica non ha una grande rilevanza nella terapia con oppiacei, la stipsi ha grande importanza. Nei confronti della depressione respiratoria, infatti, la tolleranza si sviluppa velocemente e in ogni caso prima della analgesia, ragione per la quale il paziente non ne soffre se non per errori grossolani o alle prime somministrazioni. Per la stipsi questo non succede e una volta presente accompagna il paziente per tutto il tempo della terapia. Per ripristinare il transito intestinale bisognava ricorrere a stimolanti della peristalsi come i farmaci colinergici, con scarsi risultati, o a clisteri, con risultati talora devastanti per l'equilibrio dei pazienti. Quindici anni fa [12] fa un medico geniale, che conosceva anche la farmacologia generale, somministrò ai suoi pazienti naloxone per via orale e risolse il problema. Quale è l'arcano? Il naloxone, antagonista degli oppiacei risolutivo ad esempio nelle crisi di overdose, deve essere utilizzato solo per via endovenosa perché per via orale subisce un "effetto di primo passaggio" (cioè viene metabolizzato dal fegato) del 100\%. Per questa ragione, se somminsitrato per via orale, prima di arrivare al fegato il naloxone ha modo di neutralizzare l'effetto degli oppiacei legati ai recettori intestinali, ma non riesce a raggiungere il sistema nervoso centrale e quindi a bloccare l'analgesia indotta dal legame ai recettori centrali, perché inattivato dal fegato. Dopo anni di incredulità da parte della classe medica, attualmente l'uso di antagonisti periferici per combattere la stipsi indotta dai oppiacei è pratica comune. Molte sono le alterantive: dall'uso del naloxone per via orale da solo o associato a oppiacei nella formulazione, all'impiego di naltrexone (un altro antagonista oppiaceo) modificato nella molecola per impedirne il suo passaggio al sistema nervoso centrale.

\subsection{Nuovi problemi nell'uso degli oppiacei legati al loro funzionamento}

Mentre la ricerca di base e clinica non ha portato a evidenti passi avanti nello scoprire nuove molecole, ha evidenziato nuovi problemi nell'uso degli oppiacei.

Il primo problema, legato soprattutto all'impiego nel periodo peri-operatorio, viene definito "iperalgesia da oppiacei". Si osserva una diminuzione della risposta analgesica a una dose normalmente attiva, così come nella tolleranza. In questo caso però non si tratta di tolleran- 
za, perché mentre questa è caratterizzata da una diminuzione dell'effetto del farmaco che può essere superata aumentando la dose, la iperalgesia non può essere superata aumentando la dose, bensì diminuendola, perché è una sensibilizzazione indotta dal farmaco stesso. Cosa c'è alla sua origine? Non lo sappiamo con precisione, ma è un fatto che l'iperalgesia può essere controllata con farmaci che bloccano IL1, IL6, TNFalfa, cioè quelle citochine che abbiamo detto in precedenza sono coinvolte nel sistema "dolore": un buon esempio del legame tra dolore e sistema immunitario, non legato al solo dolore neuropatico.

Un secondo problema che si è osservato nel trattamento con oppiacei è la differente risposta legata ai singoli pazienti. Siamo nel campo della farmacogenetica, cioè di tutti quei fattori legati al nostro assetto genico, che condizionano come noi ci poniamo nei confronti di un farmaco, soprattutto in termini di metabolismo e integrità dei bersagli farmacologici. Molti oppiacei sono metabolizzati a livello epatico, alcuni sono inattivati, ma altri, come la codeina, vedono aumentata la loro attività a causa del metabolismo epatico. La codeina deve la sua attività in gran parte all'essere in buona percentuale metabolizzata a morfina, che è dieci volte più potente. La codeina è un buon esempio per spiegare il possibile ruolo della farmacogenetica nella attività degli oppiacei. Se un paziente è portatore di una mutazione che causa una minore funzionalità degli enzimi epatici che metabolizzano la codeina in morfina, la codeina funzionerà meno (darà meno analgesia), gli enzimi potrebbero anche non funzionare del tutto se la mutazione producesse enzimi inattivi. Al contrario, se la mutazione risultasse in un aumento delle copie di enzima metabolizzatore, allora la codeina funzionerà più dell'atteso per quella dose. Questo potrebbe essere positivo (maggiore analgesia), ma potrebbe anche essere pericoloso. In letteratura sono descritti casi di soggetti che hanno assunto codeina come antitosse, quindi ad una dose relativamente bassa, ma sono andati in depressione respiratoria perchè la seppure piccola dose di codeina è stata tutta e rapidamente trasformata in morfina, che è molto più potente. Questo problema non riguarda solo la morfina, ma anche altri oppiacei con modalità differenti. Il tramadolo, ad esempio, è di per sé inattivo e agisce solo per mezzo di un suo metabolita prodotto a livello epatico.

Un altro problema ha per molto tempo messo gli uni contro gli altri farmacologi e terapisti del dolore. I terapisti del dolore osservavano che molte volte il paziente, improvvisamente, smetteva di rispondere all'oppiaceo che stava assumendo, ma rispondeva bene ad un altro 
oppiaceo. Per il farmacologo questo era un vero paradosso, infatti non era spiegabile che un farmaco che interagiva con un certo recettore, quando non funzionava più, potesse essere sostituito da un farmaco che interagiva con lo stesso recettore. In teoria i due farmaci erano identici. Gli oppiacei si legano a recettori specifici, che si chiamano mu, kappa, delta. Solo dopo molto tempo si è compreso che i recettori oppiacei possono essere monomeri (es. un recettore mu) o dimeri omologhi (es. un recettore $\mathrm{mu}+$ un recettore $\mathrm{mu}$ ) o dimeri eterologhi (es. un recettore $\mathrm{mu}+$ un recettore kappa) ecc. Si è visto che un farmaco può avere un effetto ottimale legandosi al monomero, ma modificare la sua attività in più o in meno se si lega a un dimero omologo o eterologo. Chi cambia quindi è il recettore che si può presentare nelle diverse forme. Quello che è curioso, e non è ancora stato spiegato, è cosa induca il diverso modo di presentarsi del recettore, per cui oggi un paziente presenta il monomero, ma improvvisamente passa a presentare un dimero omologo o no. In parallelo, farmaci oppiacei teoricamente identici come meccanismo di azione, possono essere più attivi su un monomero che su un dimero eterologo o monomero o viceversa. Questa fluidità dell'assetto recettoriale spiega perché un soggetto che smette di rispondere a un farmaco può rispondere a un farmaco teoricamente identico, ma che rispetto all'altro predilige un differente assetto recettoriale. La conclusione è stata che nella terapia con oppiacei si deve talora attuare quella che si chiama la "rotazione degli oppiacei", cioè i diversi farmaci vengono usati a rotazione nello stesso soggetto.

\subsection{Nuovi problemi nell'uso degli oppiacei legati alla valutazione del dolore}

Nel 1986, la Organizzazione Mondiale della Sanità (OMS) indicò una scala a tre "gradini" nell'intento di mettere ordine nella terapia del dolore da cancro. La scala nacque da una valutazione dell'intensità del dolore secondo la quale si doveva iniziare con i farmaci non oppiacei, per poi aggiungere oppiacei di media potenza ed infine arrivare all'uso dei farmaci più potenti come morfina o metadone. Due problemi sono sorti nell'uso di questa scala. Già poco tempo dopo la sua invenzione, ci si rese conto che i farmaci oppiacei di "media potenza" (es. codeina, tramadolo, ossicodone, buprenorfina) potevano essere tranquillamente sostituiti da basse dosi di morfina, con risultati identici e costi assolutamente inferiori. Ci si era resi conti che l'uso di questi farmaci nasceva 
più da una creazione del marketing che da un reale vantaggio terapeutico. L'OMS era tanto convinta di ciò, che definì quasi subito che i tre scalini non valevano per i paesi più poveri, dove si suggerisce di sostituire il secondo scalino con l'uso della morfina a bassi dosaggi. Direi un messaggio molto chiaro, ma inascoltato, tanto è vero che gli oppiacei di media potenza sono andati proliferando liberamente. Il problema non è risolto, ma non è scientifico o farmacologico.

Un secondo problema è nato dalla considerazione che la scala dell'OMS (a due o tre scalini) parte da una valutazione del dolore acuto, che niente o ben poco ha da spartire con il dolore cronico che, abbiamo detto, rappresenta una malattia a sé, totale e pervasiva, con aspetti anche cognitivo-comportamentali/affettivi non presenti nel dolore acuto. Recentemente sono apparsi molti articoli che sollevano questo punto $[13,14]$, ma anche in questo caso non si intravvede una soluzione. Al momento, l'unica possibilità è prendere coscienza di questo aspetto della terapia del dolore da cancro (e cronico in generale) e valutare con attenzione ogni caso, senza applicare quegli schematismi terapeutici che sembravano potere essere di aiuto, ma potevano anche portare a inevitabili insuccessi.

\subsection{Nuovi problemi nell'uso degli oppiacei legati al loro uso improprio}

L'uso degli oppiacei ha vissuto negli anni almeno tre periodi. Nell'ottocento sono stati farmaci universali, di libero consumo, usati potremmo dire con una certa "innocenza" per il dolore, le malattie più disparate e per diletto. Ben presto però questi farmaci hanno manifestato il loro aspetto peggiore che potremmo riassumere nella dipendenza e tutti i problemi sociali e medici che la accompagnano. Entriamo nel secondo periodo, che arriva fino a circa 15 anni fa, durante il quale gli oppiacei sono considerati da un lato ottimi farmaci per il dolore più grave, dall'altro sono temuti per gli effetti collaterali e il fenomeno sempre più diffuso della tossicodipendenza. Da qui una forte attenzione al loro impiego medico e regole per la loro detenzione, prescrizione e uso molto rigide e punitive: più rivolte a prevenire la tossicodipendenza che a favorire l'impiego terapeutico. Fino a qui gli oppiacei sono essenzialmente la morfina, il metadone, la codeina. Circa 15 anni fa, si è suggerito che un buon uso degli oppiacei potesse applicarsi con successo anche al dolore acuto (es. manovre diagnostiche), ma soprattutto cronico non da cancro e che in certe popolazioni, es. anziani, potessero avere 
dei vantaggi sugli analgesici più utilizzati, i FANS, (es. gli oppiacei non compromettono, a differenza dei FANS, la funzionalità renale). A questo aspetto "scientifico" della rivisitazione degli oppiacei si è presto affiancato anche un fenomeno di marketing talora molto spinto, legato alla messa in commercio di oppiacei presentati come meno "aggressivi" quali tramadolo, ossicodone, tapentadolo, in formulazioni (vedi 3.5) più agevoli da utilizzare, ma soprattutto più facilmente prescrivibili. Negli stessi anni si è fortemente sviluppata la sensibilità della comunità scientifica e non al problema dolore, fenomeno che in Italia è addirittura sfociato in una legge (!) che impone il trattamento del dolore (legge 38 del 2010). Negli stessi anni si sono diffuse alcune linee di pensiero che, su basi non sufficientemente provate, hanno suggerito che l'uso degli oppiacei finalizzato (nella mente del paziente) alla terapia del dolore lo protegga dallo svilupparsi di addiction, che solo l'uso di certi oppiacei per lungo tempo produca addiction, che solo i pazienti con certe caratteristiche vadano incontro a addiction. Il risultato di questi fattori in parte scientifici, in parte commerciali e in parte emotivi, che di fatto hanno demitizzato la pericolosità degli oppiacei, è stato che l'uso degli oppiacei è aumentato fortemente in tutto il mondo e, seppure in misura più limitata, anche in Italia.

Con quale risultato? Che ora si devono "ridurre i rischi del sollievo del dolore", come scrive il New England Journal of Medicine [15], perché le morti da overdose di oppiacei prescrivibili sono aumentate drammaticamente, 4 su 5 persone che iniziano a usare eroina, riferiscono di avere iniziato con oppiacei prescrivibili (un quadro capovolto rispetto agli anni prima del 2000) e , malgrado molti sforzi per cercare di arginare il fenomeno, non si vede alcun segno di cambiamento. Questa è la situazione attuale, ripeto non ancora così evidente da noi, ma con questo pericolo che incombe.

\subsection{Le nuove formulazioni degli oppiacei}

Tre linee di pensiero hanno guidato la ricerca di nuove formulazioni per i farmaci oppiacei: il più facile utilizzo, il controllo degli effetti collaterali, evitare l'uso di farmaci prescrivibili per usi non terapeutici (tossicodipendenza).

Le formulazioni più interessanti per facilitare l'uso degli oppiacei sono state innanzitutto quelle che hanno impiegato i cerotti per somministrazione transdermica. Queste formulazioni hanno permesso di ren- 
dere a lunga durata di azione, tre giorni, farmaci che in altro modo avrebbero avuto un'azione sì potente, ma breve come il fentanil (20 minuti). Un altro risultato è stato di ottenere una somministrazione regolare senza picchi di concentrazioni plasmatiche tali da potere portare ad effetti indesiderati (es. la buprenorfina) e da ultimo hanno permesso di evitare il passaggio dall'intestino e quindi praticamente annullare la stipsi. Un'altra formulazione interessante è stata quella in forma di "lecca-lecca" che serve a somministrare un farmaco potente, in genere il fentanil, per sedare rapidamente, pur durante una terapia già in atto, dei picchi improvvisi di dolore molto forte, quello che si chiama "dolore incidente".

Per controllare l'effetto collaterale più grave, che abbiamo detto essere la stipsi, si sono create formulazioni che contengono nella stessa compressa sia l'oppiaceo attivo che un antagonista ad attività solo periferica (es. ossicodone e naloxone), ottenendo così con un'unica somministrazione l'effetto analgesico e il blocco dell'effetto indesiderato.

Per prevenire un uso improprio degli oppiacei, cioè che le compresse potessero essere sminuzzate, sciolte in acqua e iniettate per essere usate come droga, sono state inventate delle formulazioni che, fuori dall'organismo, non possono esser né sciolte, né frantumate nemmeno prendendole a martellate (!), oppure che non possono essere trattate chimicamente in modo da estrarre dalla compressa l'oppiaceo attivo.

\section{Conclusioni}

Due e molto diverse tra loro sono le considerazioni finali che vorrei fare alla fine di questo breve contributo sul dolore e l'uso degli oppiacei.

Una è che la morfina è un farmaco che, pure con tutti i problemi che abbiamo elencato, resta il migliore analgesico per i dolori di mediagrave entità. La morfina ha solo due enormi "difetti": non è brevettabile e costa poco.

La seconda considerazione è che, come ho detto all'inizio, non credo che i farmaci analgesici e in particolare gli oppiacei, rappresentassero un interesse primario tra $\mathrm{i}$ campi di ricerca del Professor Mantegazza, ma proprio per questo devo essergli riconoscente per avermi sostenuto materialmente e umanamente nel portare avanti la mia ricerca. 


\section{BIBLIOGRAFIA}

1. Genovese E, Zonta N, Mantegazza P, Decreased antinociceptive activity of morphine in rats pretreated intraventricularly with 5,6-dihydroxytryptamine, a long-lasting selective depletor of brain serotonin, Psychopharmacologia, 1973: 32: 359-364.

2. Mantegazza P, Tammiso R, Vicentini L, Zambotti F, Zonta N, The effect of GABA ergic agents on opiate analgesia, Pharmacol Res Commun, 1980: 12: 239-247.

3. Zonta N, Zambotti F, Vicentini L, Tammiso R, Mantegazza P, Effects of some GABA-mimetic drugs on the antinociceptive activity of morphine and betaendorphin in rats, Naunyn Schmiedebergs Arch Pharmacol, 1981: 316:231-234.

4. Mantegazza P, Parenti M, Tammiso R, Vita P, Zambotti F, Zonta N, Modification of the antinociceptive effect of morphine by centrally administered diazepam and midazolam, Br J Pharmacol, 1982: 75:569-572.

5. Zambotti F, Zonta N, Parenti M, Tammiso R, Vicentini L, Conci F, Mantegazza $\mathrm{P}$, Periaqueductal gray matter involvement in the muscimol-induced decrease of morphine antinociception, Naunyn Schmiedebergs Arch Pharmacol, 1982: 318:368-379.

6. Mantegazza P, Tammiso R, Zambotti F, Zecca L, Zonta N, Purine involvement in morphine antinociception, Br J Pharmacol, 1984: 83: 883-888.

7. Zambotti F, Zonta N, Tammiso R, Conci F, Hafner B, Ferrario P, Zecca L, Mantegazza P, Zopiclone potentiates the antinociceptive effect of morphine in rats, Naunyn Schmiedebergs Arch Pharmacol, 1987: 336: 526-529.

8. Zambotti F, Zonta N, Tammiso R, Conci F, Hafner B, Zecca L, Ferrario P, Mantegazza P, Effects of diazepam on nociception in rats, Naunyn Schmiedebergs Arch Pharmacol, 1991: 344:84-89.

9. Sacerdote P, Manfredi P, Mantegazza P, Panerai AE, Antinociceptive and immunosuppressive effects of opiate drugs: a structure-related activity study, $\mathrm{Br} \mathrm{J}$ Pharmacol, 1997: 121: 834-840.

10. Sacerdote P, Franchi P, Moretti S, Castelli M, Procacci P, Magnaghi V, Panerai AE, Cytokine Modulation is Necessary for Efficacious Treatment of Experimental Neuropathic Pain, J Neuroimmune Pharmacol, 2012: DOI 10.1007/s11481-012-9428-2.

11. Stein C, The control of pain in peripheral tissue by opioids, N Engl J Med, 1995: 332: $1685-1690$.

12. Meissnera W, Schmidta U, Hartmannb M, Kathc R, Reinhar K, Oral naloxone reverses opioid-associated constipation, Pain, 2000: 84:105-109.

13. Caraceni A, Hanks G, Kaasa S, Bennett MI, Brunelli C, Cherny N, Dale O, De Conno F, Fallon F, Hanna M, Faksvåg Haugen D, Juhl G, King S, Klepstad P, Laugsand EA, Maltoni M, Mercadante S, Nabal M, Pigni A, Radbruch L, Reid C, Sjogren P, Stone PC, Tassinari D, Zeppetella G, European Palliative Care Research Collaborative (EPCRC), European Association for Palliative Care (EAPC), Use of opioid analgesics in the treatment of cancer pain: evidencebased recommendations from the EAPC, Lancet Oncol, 2012; 13: e58-68. 
14. Ballantyne JC, Kalso E, Stannard C, WHO analgesic ladder: a good concept gone astray: Our mistake is to treat chronic pain as if it were acute or end of life pain, BMJ, 2016: 352:i20 doi: 10.1136/bmj.i20.

15. Alford DP, Opioid Prescribing for Chronic Pain - Achieving the Right Balance through Education, N Engl J Med 374: 4: 301-303. 